\title{
Mean platelet volume and cardiovascular outcomes in acute myocardial infarction
}

\author{
Mangalachulli Pottammal Ranjith, ${ }^{1}$ Rajendran DivyaRaj, ${ }_{1}^{2}$ Dolly Mathew, $^{3}$ \\ Biju George, ${ }^{4}$ Mangalath Narayanan Krishnan ${ }^{3}$
}

\begin{abstract}
${ }^{1}$ Department of Cardiology, Kannur Medical College, Anjarakandy, Kerala, India ${ }^{2}$ Department of Neurology, SN Mission Hospital, Varkala, Kerala, India

${ }^{3}$ Department of Cardiology, Government Medical College, Kozhikode, Kerala, India ${ }^{4}$ Department of Community Medicine, Government Medical College, Kozhikode, Kerala, India
\end{abstract}

Correspondence to Dr Mangalachulli Pottammal Ranjith, Department of Cardiology, Kannur Medical College, P.O. Anjarakandy, Kannur-670612, Kerala, India; drranjithmp@gmail.com

Received 19 October 2015 Revised 29 December 2015 Accepted 30 December 2015

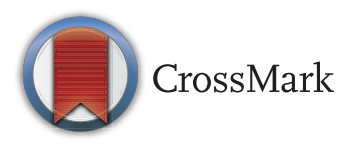

To cite: Ranjith MP, DivyaRaj R, Mathew D, et al. Heart Asia 2016;8:16-20.

doi:10.1136/heartasia-2015010696

\section{ABSTRACT}

Objective High levels of mean platelet volume (MPV)

may be associated with adverse outcomes in patients with myocardial infarction (MI). We examined the association between MPV and the risk of death and adverse cardiovascular outcomes in patients with MI.

Methods We studied consecutive patients with MI admitted to a tertiary-care hospital during a period of 1 year. MPV was measured at admission and at third month. Patients were followed up for 1-year primary composite outcome of cardiovascular death, stroke, fatal or non-fatal $\mathrm{MI}$ and cardiac failure. Patients were classified according to tertile of baseline MPV. Results A total of 1206 patients with Ml, including 934 men $(77.4 \%)$ and 272 women (22.6\%) were studied. The mean age of the study population was 56 years. At 1-year follow-up, 292 (28.57\%) primary outcome occurred: cardiovascular mortality 78 (7.6\%), fatal or non-fatal MI $153(15.0 \%)$, stroke $30(2.9 \%)$ and cardiac failure $128(12.52 \%)$. Patients with the highest tertile MPV had higher primary outcome as compared with those with MPV in the lowest tertile (adjusted $\mathrm{OR}=2.31 ; 95 \% \mathrm{Cl} 1.60$ to 3.35; $p<0.001$ ). Total mortality was also more in high-MPV group (adjusted OR 2.62; $95 \% \mathrm{Cl} 1.47$ to $4.70 ; p<0.001)$. There were no significant changes in mean MPV values at admission from those at third month interval $(9.15$, (SD 0.99) vs 9.19 (SD 0.94); $p=0.2$ ).

Conclusions Elevated MPV was associated with worse outcome in patients with acute MI. Elevated MPV in these patients may be due to inherently large platelets. Trial registration number http://ctri.nic.in/ Clinicaltrials/rmaindet.php?trialid=5485\&EncHid=98036. 61144\&modid=1\&compid=19; CTRI/2012/12/003222.

\section{INTRODUCTION}

Mean platelet volume (MPV) is an indicator of mean size of the platelet population. Activated platelets have an important role in the initiation of atherosclerotic lesions and subsequent complications. ${ }^{1}$ Previous studies have shown MPV as an independent variable for prognosis in patients with cardiovascular diseases such as myocardial infarction (MI), heart failure or stroke, and also in patients undergoing coronary bypass surgery or coronary angioplasty. ${ }^{2-9}$ Other studies have suggested increased MPV as an indicator of larger, more reactive platelets resulting from an increased platelet turnover as potential mechanism linking MPV to outcome. ${ }^{10} 11$ But a few studies have shown no correlation between MPV and prognosis in MI. ${ }^{12}$ There are conflicting reports regarding the prognostic value of $\mathrm{MPV}$ in $\mathrm{MI}$; there are no studies assessing the prognostic value of MPV in
MI among Asian population. We tested the hypothesis that higher levels of MPV were associated with risk of all-cause mortality and adverse cardiovascular outcomes in a population with MI.

\section{METHODS}

\section{Study design and patients}

In a single-centre prospective cohort study, we included consecutive patients admitted to the coronary care unit of a large tertiary hospital with a diagnosis of non-ST-elevation MI (NSTEMI) and ST-elevation MI (STEMI) from December 2011 to November 2012. The American College of Cardiology diagnostic criteria were used for making the diagnosis of MI. ${ }^{13}$ Hypertension was defined as systolic blood pressure $\geq 140 \mathrm{~mm} \mathrm{Hg}$, diastolic blood pressure $\geq 90 \mathrm{~mm} \mathrm{Hg}$ or current therapy for hypertension. Dyslipidaemia was defined as a serum total cholesterol concentration $\geq 220 \mathrm{mg} / \mathrm{dL}$, low-density lipoprotein cholesterol concentration $\geq 140 \mathrm{mg} / \mathrm{dL}$ or ongoing lipid-lowering therapy. Patients whose platelet indices could not be estimated within $12 \mathrm{~h}$ of presentation or before revascularisation were excluded. Patients with previous MI in 6 months or with history of bleeding disorders, pre-eclampsia, sepsis, thrombocytopaenia, blood transfusion or major operation or trauma during the last 3 months, patients who were receiving drugs that could cause thrombocytopaenia were also excluded. Patients were followed up for a period of 1 year by telephonic interview; those who had a suspected outcome event were reviewed by examining hospital admission reports or during outpatient visits.

The ethics committee of study centre approved the study in accordance with ethical standards of the Declaration of Helsinki, and all patients gave their informed consent. Full clinical evaluation was performed. Baseline investigations included complete blood count, blood sugar, serum urea and creatinine, serum lipid profile, serum troponin-I and creatine phosphokinase myocardial band (CPK$\mathrm{MB})$, ECG and comprehensive echocardiography. Troponin I >0.11 ng/L and CPK-MB >7.0 $\mu \mathrm{g} / \mathrm{L}$ were taken as positive. Estimation of platelet count and MPV was performed in all patients. Venous blood samples were drawn from all subjects after admission (within $30 \mathrm{~min}$ ) before initiation of treatment. For sample collection, standardised EDTA sample tubes were used, and all samples were processed within 30 min after blood collection, using a Sysmex KX21-N autoanalyser (Sysmex, Kobe, Japan). 


\section{Study outcomes}

The primary outcome for this study was a composite of cardiovascular mortality, stroke, non-fatal MI and cardiac failure requiring hospitalisation at 1-year follow-up. All deaths were considered as cardiac unless a clear non-cardiac cause could be established. Secondary outcomes were inhospital mortality, fatal or non-fatal MI, the development of symptomatic heart failure, ischaemic or non-ischaemic stroke and cardiovascular mortality at 1 year. MI and stroke were defined according to the American Heart and Stroke Association criteria. Patients admitted with a discharging diagnosis of heart failure were counted as cardiac failure event.

\section{Statistical analysis}

The continuous variables were presented as means and SDs. Categorical variables were presented as percentages. Patients were divided into subgroups according to MPV tertile. The cut-offs for tertile taken were 33.33 and 66.66 percentiles of baseline MPV values. Group I $(n=434)$ comprised patients with an MPV $\leq 8.7 \mathrm{fL}$ (tertile 1), group II $(\mathrm{n}=406)$ comprised patients with an MPV value of $>8.7 \mathrm{fL}$ and $\leq 9.5 \mathrm{fL}$ (tertile 2) and group III $(n=366)$ comprised patients with MPV values of $>9.5 \mathrm{fL}$ (Tertile 3 ). To test for differences across all groups, the $\chi^{2}$ and one-way analysis of variance tests were used. Multivariate regression analysis was done and variables which were likely to affect the outcome were included in the model. Outcome was adjusted for age, dyslipidaemia, stroke, hypertension, coronary artery disease, diabetes, history of antiplatelet consumption, smoking, ejection fraction $<50 \%$ and creatinine. Analyses were performed with the SPSS statistical software (V.18.0; SPSS, Chicago, Illinois, USA).

\section{RESULTS}

\section{Baseline characteristics}

A total of 1206 patients with MI including 934 men (77.4\%) and 272 women $(22.6 \%)$ were studied. The mean age of the study population was 56 years. Among the population, 797 $(66.09 \%)$ were diagnosed as STEMI and rest of them as NSTEMI. The MPV ranged from $6.3 \mathrm{fL}$ to $13.2 \mathrm{fL}$. (Median 9.1 fL; mean 9.17(SD 1.0 fL) and 162 (13.4\%) had MPV levels outside the normal range of $8-11 \mathrm{fL}$ with $\mathrm{MPV}<8 \mathrm{fL} \quad(\mathrm{n}=108$
$(8.96 \%))$ and $>11 \mathrm{fL}(\mathrm{n}=54(4.48 \%)$.) Among the total population, $84.7 \%$ completed 1-year follow-up (first tertile $86.2 \%$, second tertile $79.8 \%$, third tertile $88.5 \%$; p for trend 0.453 ). There was no significant difference in MPV values between those patients taking antiplatelets and those not taking $(9.29 \pm 1$ vs $9.15 \pm 0.99 ; p=0.082)$. The baseline characteristics of patients are shown in table 1 .

\section{Association between MPV and primary outcome}

At 1-year follow-up, primary outcome occurred in 292 $(28.57 \%)$ of which cardiovascular mortality comprised of 78 (7.6\%), fatal or non-fatal MI in $153(15.0 \%)$, stroke in 30 (2.9\%) and cardiac failure in $128(12.52 \%)$. When participants were divided into three categories on the basis of tertile of baseline MPV level, a graded relation between MPV and primary outcome was observed $(p=0.01)$. Participants with MPV in the highest tertile had fully adjusted OR for experiencing the primary outcome of 1.71 (95\% CI 1.18 to 2.45) compared with those with MPV in the lowest tertile (table 2, figure 1). When the second and third tertiles of MPV were compared similar results were obtained (adjusted OR-1.76; 95\% CI 1.22 to 2.56; $\mathrm{p}=0.003)($ table 3)

\section{Association between MPV and cardiovascular mortality}

The incidence of cardiovascular mortality was $7.6 \%$ among those who completed 1 year of follow-up. There was a graded relation between MPV and cardiovascular mortality at 1-year follow-up among the MPV tertile groups $(p=0.001)$. For example, the fully adjusted risk of cardiovascular mortality in the highest category of MPV was 2.83 (95\% CI 1.49 to 5.35) compared with the referent group and 2.85 (95\% CI 1.5 to 5.41) as compared with second tertile (tables 2 and 3 ).

\section{Association between MPV and secondary outcome}

Higher levels of MPV were associated with an increased risk of fatal or non-fatal MI after adjustment for different clinical variables $(p=0.025)$. Participants with MPV in the highest tertile had fully adjusted OR for developing fatal or non-fatal MI of 1.91 (95\% CI 2.2 to 3.04) compared with those with MPV in the lowest tertile. Inhospital mortality, incidence of new symptomatic heart failure and stroke were high in the third tertile

Table 1 Baseline characteristics of study population

\begin{tabular}{|c|c|c|c|c|}
\hline & MPV tertile $1(n=434)$ & MPV tertile $2(n=406)$ & MPV tertile $3(n=366)$ & p Value \\
\hline Age, mean (SD) & $55(10.87)$ & $57(10.87)$ & $56(11.46)$ & 0.06 \\
\hline Men & $336(77.4)$ & $326(80.3)$ & $272(74.3)$ & 0.14 \\
\hline Dyslipidaemia & $81(18.7)$ & $110(27.1)$ & $94(25.7)$ & 0.01 \\
\hline Diabetes & $120(27.6)$ & $124(30.5)$ & $118(32.2)$ & 0.35 \\
\hline Hypertension & $134(30.9)$ & $144(35.5)$ & $140(38.3)$ & 0.08 \\
\hline Stroke & $22(5.1)$ & $18(4.4)$ & $14(3.8)$ & 0.70 \\
\hline Coronary artery disease & $48(11.1)$ & $40(9.9)$ & $48(13.1)$ & 0.35 \\
\hline Current smoker & $180(41.5)$ & $188(46.3)$ & $130(35.5)$ & 0.01 \\
\hline History of antiplatelet consumption & $60(13.8)$ & $55(13.5)$ & 67 (18.3) & 0.12 \\
\hline Platelet count $\left(\times 10^{9} / \mathrm{L}\right)$, mean $(\mathrm{SD})$ & $259.39(66.23)$ & $238.69(57.37)$ & $213.50(65.09)$ & $<0.001$ \\
\hline Total cholesterol (mg/dL), mean (SD) & $188.58(53.60)$ & $181.44(36.82)$ & $186.48(43.09)$ & 0.08 \\
\hline Triglycerides (mg/dL), mean (SD) & 111.35 (44.94) & $115.46(44.84)$ & $122.12(44.09)$ & 0.005 \\
\hline LDL cholesterol (mg/dL), mean (SD) & $121.21(44.96)$ & $118.29(71.70)$ & $119.35(40.46)$ & 0.76 \\
\hline Creatinine (mg/dL), mean (SD) & $1.06(0.33)$ & $1.09(0.39)$ & $1.18(0.58)$ & $<0.001$ \\
\hline Ejection fraction (\%), mean (SD) & $57.50(10.74)$ & $57.01(10.15)$ & $58.12(10.60)$ & 0.35 \\
\hline
\end{tabular}




\section{Original research}

Table 2 Adjusted association between tertile of MPV and clinical outcomes

\begin{tabular}{|c|c|c|c|c|c|c|c|}
\hline & \multicolumn{4}{|l|}{ Unadjusted } & \multicolumn{3}{|c|}{ Adjusted* } \\
\hline & Events, n (\%) & OR & $95 \% \mathrm{Cl}$ & p Value & OR & $95 \% \mathrm{Cl}$ & p Value \\
\hline \multicolumn{4}{|c|}{ Primary outcome } & $0.001 \dagger$ & & & $<0.001$ \\
\hline Tertile 1 & $92(24.6)$ & 1 & & & 1 & & \\
\hline Tertile 2 & $84(25.9)$ & 1.28 & 0.86 to 1.90 & & 1.31 & 0.89 to 1.95 & \\
\hline Tertile 3 & $116(35.8)$ & 2.26 & 1.57 to 3.24 & & 2.31 & 1.60 to 3.35 & \\
\hline \multicolumn{4}{|c|}{ Cardiovascular mortality at 1 year } & $<0.001 \dagger$ & & & $<0.001$ \\
\hline Tertile 1 & $20(5.3)$ & 1 & & & 1 & & \\
\hline Tertile 2 & $16(4.9)$ & 0.92 & 0.47 to 1.81 & & 0.92 & 0.46 to 1.86 & \\
\hline Tertile 3 & $42(13.0)$ & 2.64 & 1.51 to 4.59 & & 2.62 & 1.47 to 4.70 & \\
\hline \multicolumn{4}{|c|}{ Inhospital mortality } & $0.096 \dagger$ & & & 0.196 \\
\hline Tertile 1 & $8(1.8)$ & 1 & & & 1 & & \\
\hline Tertile 2 & $10(2.5)$ & 1.35 & 0.53 to 3.44 & & 1.51 & 0.57 to 3.98 & \\
\hline Tertile 3 & $16(4.4)$ & 2.43 & 1.03 to 5.76 & & 2.26 & 0.92 to 5.52 & \\
\hline \multicolumn{4}{|c|}{ Fatal or non-fatal myocardial infarction } & $0.01+$ & & & 0.01 \\
\hline Tertile 1 & $41(11.0)$ & 1 & & & 1 & & \\
\hline Tertile 2 & $51(15.7)$ & 1.38 & 0.89 to 2.13 & & 1.29 & 0.83 to 2.00 & \\
\hline Tertile 3 & $61(18.8)$ & 1.92 & 1.26 to 2.93 & & 1.92 & 2.25 to 2.95 & \\
\hline \multicolumn{4}{|c|}{ New symptomatic heart failure } & $0.542 \dagger$ & & & 0.41 \\
\hline Tertile 1 & $45(12)$ & 1 & & & 1 & & \\
\hline Tertile 2 & $39(12)$ & 0.92 & 0.59 to 1.44 & & 0.85 & 0.54 to 1.36 & \\
\hline Tertile 3 & $44(13.6)$ & 1.18 & 0.76 to 1.84 & & 1.18 & 0.74 to 1.86 & \\
\hline \multicolumn{4}{|l|}{ Stroke } & $0.12 \dagger$ & & & 0.183 \\
\hline Tertile 1 & $10(2.7)$ & 1 & & & 1 & & \\
\hline Tertile 2 & $6(1.9)$ & 0.64 & 0.23 to 1.78 & & 0.66 & 0.24 to 1.87 & \\
\hline Tertile 3 & $14(4.3)$ & 1.69 & 0.74 to 3.84 & & 1.61 & 0.69 to 3.75 & \\
\hline
\end{tabular}

MPV group as compared with first tertile $(4.4 \%$ vs $1.8 \%$, $13.6 \%$ vs $12 \%, 4.3 \%$ vs $2.7 \%$ ). However, after adjustment for clinical variables, this difference was not statistically significant (table 2).

\section{Relation of MPV with baseline characteristics}

The MPV value repeated at third month did not show significant changes from baseline MPV values (mean; 9.15 \pm 0.99 vs $9.19 \pm 0.94 ; p=0.2$ ). There was a significant correlation between age and MPV values $(r=0.69 ; p=0.016)$. Even though weak, serum creatinine also showed a positive correlation with MPV $(\mathrm{r}=0.131 ; \mathrm{p}<0.001)$. There was a negative correlation between MPV and platelet count $(\mathrm{r}=-0.339 ; \mathrm{p}<0.001)$ (table 4).

\section{DISCUSSION}

Larger platelets, characterised by high MPV, was associated with increased platelet reactivity which represents a major risk factor for atherothrombosis. ${ }^{14}$ Several studies have found that high

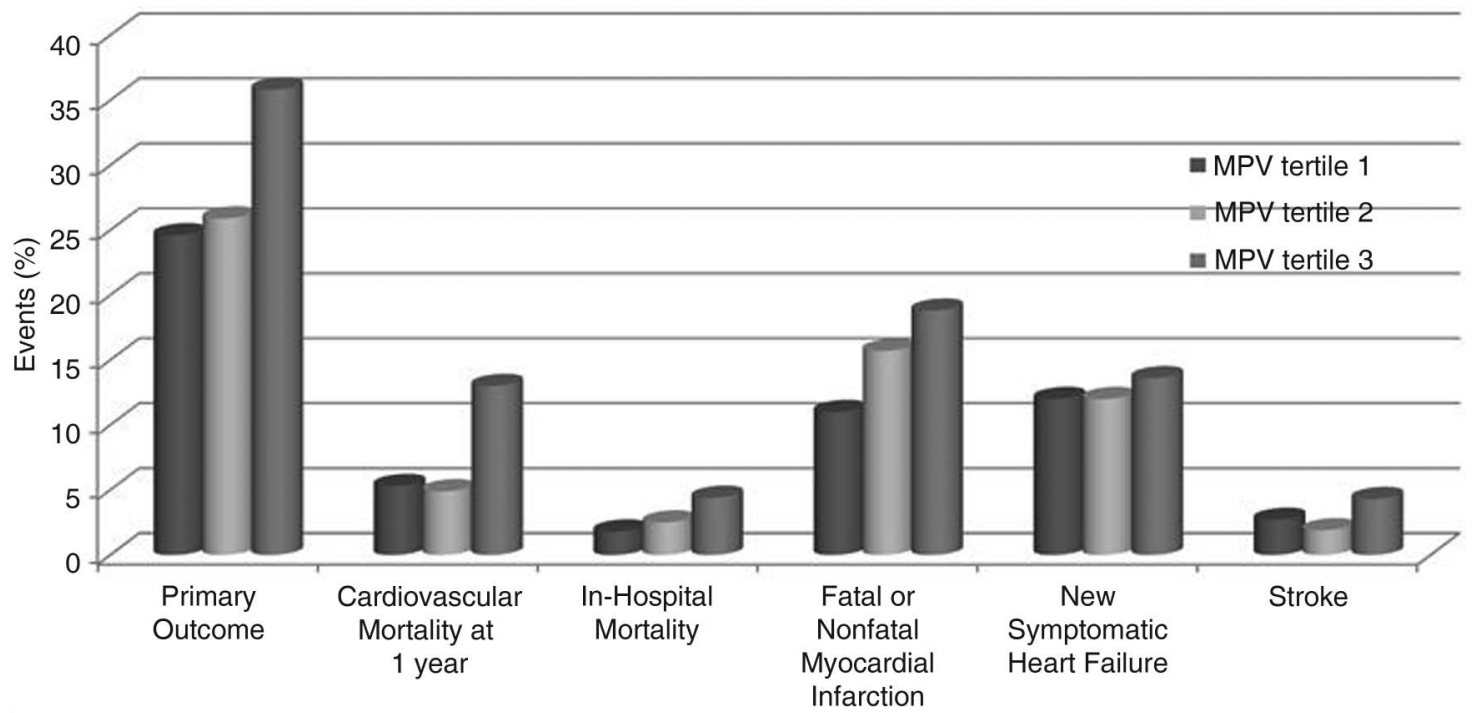

Figure 1 Association of mean platelet volume (MPV) with various cardiovascular events. 
Table 3 Adjusted association between second and third tertile of MPV and clinical outcomes

\begin{tabular}{|c|c|c|c|c|c|c|c|}
\hline & \multicolumn{4}{|l|}{ Unadjusted } & \multicolumn{3}{|c|}{ Adjusted* } \\
\hline & Events, n (\%) & OR & $95 \% \mathrm{Cl}$ & p Value & OR & $95 \% \mathrm{Cl}$ & p Value \\
\hline \multicolumn{4}{|c|}{ Primary outcome } & $0.002 \dagger$ & & & 0.003 \\
\hline Tertile 2 & $84(25.9)$ & 1 & & & 1 & & \\
\hline Tertile 3 & $116(35.8)$ & 1.75 & 1.22 to 2.51 & & 1.76 & 1.22 to 2.56 & \\
\hline \multicolumn{4}{|c|}{ Cardiovascular mortality at 1 year } & $0.001 \dagger$ & & & 0.001 \\
\hline Tertile 2 & $16(4.9)$ & 1 & & & 1 & & \\
\hline Tertile 3 & $42(13.0)$ & 2.87 & 1.58 to 5.21 & & 2.85 & 1.5 to 5.41 & \\
\hline \multicolumn{4}{|c|}{ Inhospital mortality } & $0.147 \dagger$ & & & 0.36 \\
\hline Tertile 2 & $10(2.5)$ & 1 & & & 1 & & \\
\hline Tertile 3 & $16(4.4)$ & 1.81 & 0.81 to 4.04 & & 1.50 & 0.63 to 3.56 & \\
\hline \multicolumn{4}{|c|}{ Fatal or non-fatal myocardial infarction } & $0.107 \dagger$ & & & 0.046 \\
\hline Tertile 2 & $51(15.7)$ & 1 & & & 1 & & \\
\hline Tertile 3 & $61(18.8)$ & 1.39 & 0.93 to 2.08 & & 1.52 & 1.01 to 2.3 & \\
\hline \multicolumn{4}{|c|}{ New symptomatic heart failure } & $0.28 t$ & & & 0.129 \\
\hline Tertile 2 & $39(12)$ & 1 & & & 1 & & \\
\hline Tertile 3 & $44(13.6)$ & 1.29 & 0.82 to 2.03 & & 1.45 & 0.90 to 2.34 & \\
\hline \multicolumn{4}{|l|}{ Stroke } & $0.048 t$ & & & 0.074 \\
\hline Tertile 2 & $6(1.9)$ & 1 & & & 1 & & \\
\hline Tertile 3 & $14(4.3)$ & 2.65 & 1.01 to 6.97 & & 2.44 & 0.92 to 6.51 & \\
\hline
\end{tabular}

MPV levels were associated with overall increased vascular mortality and other cardiovascular events, including MI. ${ }^{5} \quad 15 \quad 16$ Therefore, MPV has been suggested as a simple marker of functional status of platelets and may represent a risk factor for vascular adverse events. Our study found a graded, independent association between baseline MPV level and the risk of a composite of cardiovascular mortality, stroke, fatal or non-fatal MI and cardiac failure. This association was primarily driven by fatal or non-fatal MI and cardiovascular mortality at 1 year, while symptomatic heart failure, ischaemic or non-ischaemic stroke did not independently show any association with MPV values. Also, MPV did not show any significant independent association with inhospital mortality.

Adjustment for multiple potential confounders did not eliminate the association between higher MPV levels and the occurrence of primary outcome, cardiovascular mortality or reinfarction. In addition to reaching statistical significance, the magnitude of the increased risk associated with higher levels of MPV was clinically relevant. For example, the risk of cardiovascular mortality at 1 year in participants with MPV in the highest tertile was nearly $120 \%$ higher than those in the lowest tertile. This was comparable with the adjusted risk of recurrent cardiovascular events among Cholesterol and Recurrent Events study participants with high and low $\mathrm{C}$ reactive protein. ${ }^{17}$ These findings are notable given that MPV is widely available to clinicians as part of the complete blood count and therefore incurs no additional cost, in contrast to other novel markers of cardiovascular risk.

Many studies have shown a negative correlation between platelet count and MPV, and no relation between MPV and antiplatelet use. $^{3} 1819$ These results were replicated in our study, but our study showed a positive correlation between MPV and age or serum creatinine.

The mechanisms for an increased platelet volume are not fully understood. There are several theories that may explain the increase in MPV in acute coronary syndrome (ACS). One explanation for this is the consumption of platelets and release of larger and more immature platelets from bone marrow in to the circulation. ${ }^{20}{ }^{21}$ Another hypothesis is that in some patients, platelets are larger and metabolically more active and hence predisposing them for ACS. But if the increase in platelet volume is a transient phenomenon occurring during ACS, it has to normalise by few months after the event. In our study, when the MPV was repeated after 3 months, there was no significant change from the baseline.

Strengths of our analysis include relatively large sample size and the use of a central laboratory for all assays. However, we could not exclude the presence of heterotypic platelet aggregates in the high-MPV group as such phenomenon would only be determined by flow cytometry, which is currently costly, timeconsuming and needs specialised equipment. ${ }^{22}$ Previous studies have reported that MPV increases in a time-dependent manner when EDTA is used as an anticoagulant. ${ }^{23}$ However, a more recent study demonstrated that this increase of platelet size amounts to approximately $0.5 \mathrm{fL}$ when the analysis was performed within $2 \mathrm{~h}$ after sample collection. ${ }^{24}$ The reported platelet swelling with EDTA may have been due to varying concentrations of EDTA used in the blood tubes; therefore to minimise the effect of EDTA on platelet size in the present study, standardised sample tubes were used, and all samples were processed within $30 \mathrm{~min}$ after blood collection.

Table 4 Correlation between mean platelet volume and other variables

\begin{tabular}{lrr}
\hline & $r$ & $p$ Value \\
\hline Age & 0.69 & 0.016 \\
Platelet count & -0.339 & $<0.001$ \\
Serum creatinine & 0.131 & $<0.001$ \\
\hline
\end{tabular}




\section{CONCLUSION}

The current study found that MPV values at admission is a strong and independent predictor of composite of cardiovascular mortality, stroke, fatal or non-fatal MI and cardiac failure at 1-year follow-up among patients with MI. This effect was primarily driven by occurrence of cardiovascular mortality and reinfarction. Elevated MPV in these patients may be due to inherently large platelets.

\section{Key messages}

\section{What is already known about this subject?}

Mean platelet volume (MPV) is an indicator of mean size of the platelet population. Activated platelets have an important role in the initiation of atherosclerotic lesions and subsequent complications. The association between MPV and prognosis has been studied in patients with coronary artery disease. There are conflicting reports regarding the prognostic value of MPV in myocardial infarction (MI).

\section{What does this study add?}

This study found a graded, independent association between baseline MPV level and the risk of composite of cardiovascular mortality; stroke, fatal or non-fatal MI and cardiac failure at 1-year follow-up among patients with MI. Elevated MPV in these patients may be due to inherently large platelets.

\section{How might this impact on clinical practice?}

Stratification using MPV helped to predict the adverse cardiac events in patients with MI. So, MPV can be used as a prognostic indicator in Asian population with MI.

Contributors All the authors contributed to planning, conducting of the study and preparing the article for publication.

Competing interests None declared.

Ethics approval Medical College, Kozhikode, Ethics Committee.

Provenance and peer review Not commissioned; externally peer reviewed.

\section{REFERENCES}

1 Massberg S, Schulz C, Gawaz M. Role of platelets in the pathophysiology of acute coronary syndrome. Semin Vasc Med 2003;3:147-62.

2 Ranjith MP, Divya R, Mehta VK, et al. Significance of platelet volume indices and platelet count in ischaemic heart disease. J Clin Pathol 2009;62;830-3.

3 Yilmaz MB, Cihan G, Guray $Y$, et al. Role of mean platelet volume in triaging acute coronary syndromes. J Thromb Thrombolysis 2008:26:49-54.

4 Burr ML, Holliday RM, Fehily AM, et al. Haematological prognostic indices after myocardial infarction: evidence from the diet and reinfarction trial (DART). Eur Heart J 1992;13:166-70.
5 Georg S, Thomas $\mathrm{P}$, Helmuth $\mathrm{H}$, et al. Mean platelet volume may represent a predictive parameter for overall vascular mortality and ischemic heart disease. ArteriosclerThrombVasc Biol 2011;31:1215-18.

6 Greisenegger S, Endler G, Hsieh $\mathrm{K}$, et al. Is elevated mean platelet volume associated with a worse outcome in patients with acute ischemic cerebrovascular events? Stroke 2004;35:1688-91.

7 Binita S, Brandon O, Lakshmi T, et al. Mean platelet volume is significantly associated with survival following percutaneous coronary intervention and coronary artery bypass surgery [Meeting Abstract]. J Am Coll Cardiol 2012;59:E1478.

8 Tomasz R, Aleksandra J, Jakub F, et al. Prognostic value of platelet indices after acute myocardial infarction treated with primary percutaneous coronary intervention. Cardiol J 2013;20:491-8.

9 Khandekar MM, Khurana AS, Deshmukh SD, et al. Platelet volume indices in patients with coronary artery disease and acute myocardial infarction: an Indian scenario. J Clin Pathol 2006;59:146-9.

10 Pereg D, Berlin T, Mosseri M. Mean platelet volume on admission correlates with impaired response to thrombolysis in patients with ST-elevation myocardial infarction. Platelets 2010;21:117-21.

11 Martin JF, Trowbridge EA, Salmon G, et al. The biological significance of platelet volume: its relationship to bleeding time, platelet thromboxane B2 production and megakaryocyte nuclear DNA concentration. Thromb Res 1983:32:443-60.

12 Ihara A, Kawamoto T, Matsumoto K, et al. Relationship between hemostatic factors and the platelet index in patients with ischemic heart disease. Pathophysiol Haemost Thromb 2006;35:388-91.

13 Braunwald E, Antman EM, Beasley JW, et al. ACC/AHA guidelines for the management of patients with unstable angina and non-ST-segment elevation myocardial infarction. A report of the American College of Cardiology/American Heart Association Task Force on Practice Guidelines (Committee on the Management of Patients With Unstable Angina). J Am Coll Cardiol 2000;36:970-1062.

14 Kamath S, Blann AD, Lip GY. Platelet activation: assessment and quantification. Eur Heart J 2001;22:1561-71.

15 Boos CJ, Balakrishnan B, Lip GY. The effects of coronary artery disease severity on time-dependent changes in platelet activation indices in stored whole blood. J Thromb Thrombolysis 2008;25:135-40.

16 Yilmaz MB, Cihan G, Guray Y, et al. Role of mean platelet volume in triagging acute coronarysyndromes. J Thromb Thrombolysis 2008;26:49-54.

17 Ridker PM, Rifai N, Pfeffer MA, et al. Cholesterol and Recurrent Events (CARE) Investigators. Inflammation, pravastatin, and the risk of coronary events after myocardial infarction in patients with average cholesterol levels. Circulation 1998;98:839-44.

18 Erhart S, Beer JH, Reinhart WH. Influence of aspirin on platelet count and volume in humans. Acta Haematol 1999;101:140-4.

19 Harrison P, Segal H, Keeling D. 'Aspirin resistance' detected by high shear platelet function testing within the pilot phase of the Oxford Vascular Study(OXVASC) [abstract]. J Thromb Haemost 2003;1(Suppl 1):757.

20 Ault KA, Rinder HM, Mitchell J, et al. The significance of platelets with increased RNA content (reticulated platelets). A measure of the rate of thrombopoiesis. Am J Clin Pathol 1992;98:637-46.

21 Sewell R, Ibbotson RM, Phillps R, et al. High mean platelet volume after myocardial infarction: is it due to consumption of small platelets? $\mathrm{Br}$ Med J 1984:289:1576-8.

22 Gibson CM, Murphy SA, Rizzo MJ, et al. Relationship between TIMI frame count and clinical outcomes after thrombolytic administration. Thrombolysis In Myocardial Infarction (TIMI) Study Group. Circulation 1999;99: 1945-50.

23 Bath PM, Butterworth RJ. Platelet size: measurement, physiology and vascular disease. Blood Coagul Fibrinolysis 1996;7:157-61.

24 Endler G, Klimesch A, Sunder-Plassmann H, et al. Mean platelet volume is an independent risk factor for myocardial infarction but not for coronary artery disease. Br J Haematol 2002;117:399-404. 